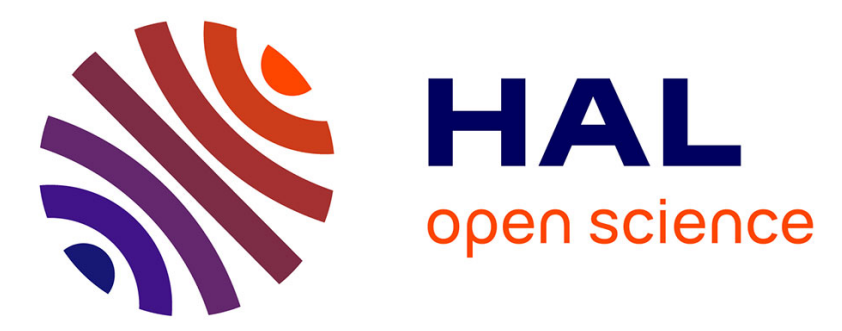

\title{
Phantom Sensation: When the phantom escapes the bounds of the actuators and the end-point is sensed in the air
}

Angelina Bellicha, Andres Trujillo-Leon, Wael Bachta

\section{- To cite this version:}

Angelina Bellicha, Andres Trujillo-Leon, Wael Bachta. Phantom Sensation: When the phantom escapes the bounds of the actuators and the end-point is sensed in the air. 2019 IEEE World Haptics Conference (WHC), Jul 2019, Tokyo, France. pp.91-96, 10.1109/WHC.2019.8816176 hal-02492269

\section{HAL Id: hal-02492269 \\ https://hal.sorbonne-universite.fr/hal-02492269}

Submitted on 26 Feb 2020

HAL is a multi-disciplinary open access archive for the deposit and dissemination of scientific research documents, whether they are published or not. The documents may come from teaching and research institutions in France or abroad, or from public or private research centers.
L'archive ouverte pluridisciplinaire HAL, est destinée au dépôt et à la diffusion de documents scientifiques de niveau recherche, publiés ou non, émanant des établissements d'enseignement et de recherche français ou étrangers, des laboratoires publics ou privés. 


\title{
Phantom Sensation: When the phantom escapes the bounds of the actuators and the end-point is sensed in the air
}

\author{
Angélina Bellicha, Andrés Trujillo-León and Wael Bachta
}

\begin{abstract}
The Phantom Sensation is a case of spatiotemporal interaction. In this paper, we study the influence of the duration of a stimulation on the position of the perceived end-point of the illusion. We find that the position of the end-point is highly dependent on the duration of the stimulation. Three experiments have been conducted. Participants held an object delivering phantom sensations of different durations. We show that for durations ranging from 0.25 to $2.5 \mathrm{~s}$, the direction of the illusory motion is well perceived (experiment 1). We secondly show that for increased durations $(>0.75 s)$, the perceived endpoint of the illusion can be located outside of the hand, inside the object held (experiment 2). We finally show that for increased durations $(>1.5 \mathrm{~s})$, the perceived end-point can be located even further, outside of the object held, i.e. beyond the intra-actuator space (experiment 3).
\end{abstract}

\section{INTRODUCTION}

The tactile modality is widely used in human machine interfaces as it is an easy and safe way to convey information without hindering the other senses.

Conveying tactile information can be based on tactile illusions of motion, which have been studied since the beginning of the last century [1]. A tactile illusion is usually described as a discrepancy between the expected perception of a physical stimulus and the real perception, that is usually surprising and perplexing [2].

In his paper [1], Burtt found that, under some conditions of intensity, spacing and time, participants could perceive a motion between two tactile stimuli. Whether the perceived motion is discrete (the rabbit illusion [3], [4]) or continuous (the phantom sensation [5]), it can convey an information of direction [6].

During Phantom Sensations (PS), spatiotemporal interactions are at play. Alles suggests in [5] that the PS may arise from a combination of temporal and amplitude inhibitions. First, he explains that two stimuli of equal amplitude, occurring subsequently with a short time delay will be perceived as one stimulus. The perceived position depends on the time delay, often called Inter-Stimuli-Onset-Interval (ISOI) [7]. Then, he explains that two stimuli of varying amplitude are perceived as a motion between them. The perceived position is hence modified by a change of amplitude through time. In short, there is a close relationship between the perception of distance and time [8].

Berger et al. [9] explain that the perceived area of stimulation is not always at the location of the physical contact with

\footnotetext{
*This work was supported by the ANR within the project i-Gait, under reference ANR-16-CE33-0012

All the authors are members of the ISIR laboratory, Agathe Group, UMR 7222, CNRS, Sorbonne Université, UI-5110 Inserm, Paris, France \{bellicha, trujillo,bachta\}eisir.upme.fr
}

the skin. In their experiment, participants were holding two handles, one in each hand, while seeing their hands only holding a single object through a Virtual Reality Display. Participants felt a motion going from one hand to the other. Other experiments confirm that a tactile stimulation can be perceived out of the body between the stimulated parts of the body ([10],[9],[11]). Miyazaki et al. explain in [10] this mislocalisation outside of the body by saying that the object is added to the mental scheme of the body. In all these studies on the mislocalisation, the end-point of the illusion never goes beyond the area defined by the actuators.

The PS described in [5] is an interesting case of spatiotemporal interaction. Moreover, this illusion is reported to have a good direction detection success rate. It could be more useful in applications such as assistance, rehabilitation or virtual reality systems if the perceived end-point of the movement could be controlled.

However, Alles in [5] found that depending on the displayed PS, the end-point of the PS cannot be perceived easily, if not at all, by participants. This comment in [5] was the start point of our study on the perception of the end-point of PS illusions.

We conducted a preliminary investigation of the perception of the end-point of a PS. This investigation aims to study whether the end-point of a PS could be perceived out of the hand or even outside of the object held.

Three experiments have been conducted. The experiment 1 "Discrimination of the direction" shows that the direction detection is independent of the stimulation duration. The experiment 2 "Out of hand detection" shows that the endpoint of the PS can be perceived out of the hand palm. The experiment 3 "Out of object detection" shows that the endpoint could even be perceived out of a grasped object, in the air.

To this end, two custom handles of different lengths were used. Both handles are fitted with two actuators each. The distance inter-actuator is the same for the two handles and approximately equal to an average hand palm width. The duration of the PS was varied, but the maximal amplitude of stimulation was the same across durations.

In this paper, we show that a tactile stimulation can be perceived out of the hand, in the object or in the air beyond the object which is beyond the stimulators positions. These mislocalisations seem related to the duration of the tactile illusions. 


\section{MATERIAL AND METHODS}

\section{A. Experimental setup}

The experimental setup is based on two custom handles of different lengths. The long handle ( $345 \mathrm{~mm}$ long) is depicted in Fig 2. The short handle (of $143 \mathrm{~mm}$ long) is in Fig 3.

Both handles consist in a T-shape support composed of equal halves, spaced by $1 \mathrm{~mm}$. Two actuators vibrating tangentially are embedded into each half, using silicon material. Spacing and the use of silicon are meant to reduce interferences between the actuators. The actuators are placed $7 \mathrm{~cm}$ apart. This distance corresponds roughly to the mean width of a hand palm. The actuators have been built based on the recommendation of [12].They are replicable.

The long handle is meant to help us determine whether an out of hand end-point perception is possible.

The short handle was designed to see whether an out of object end-point perception is possible. Its length has been defined carefully. The length was chosen to be comfortably grasped, and avoid participants determining its length during the experiment.

The experimental setup schematized in Fig. 1 explains the driving of the actuators of both handles.

The setup works as follows: MATLAB is used to generate the two signals that drive the vibrotactile actuators creating the tactile illusion. Outputs of the NI USB6001 card, running at a $2 \mathrm{kHz}$ rate are finally passed through audio-amplifiers before being sent to the actuators.

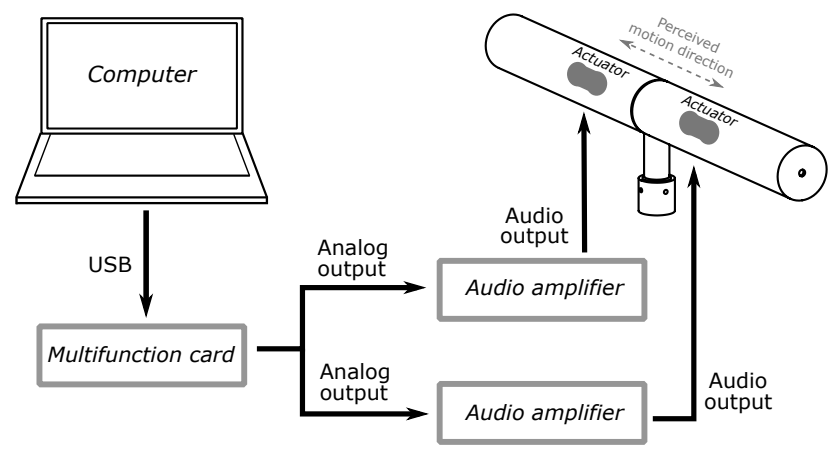

Fig. 1. Experimental setup scheme.

For experiments 2 and 3, 2 sheets of paper were used. The drawing in Fig. 4(a) is the participant sheet. It represents the long handle. The gray area represents their grasp on the handle. The drawing shown in Fig. 4(b) is the experimenter sheet. The drawing is divided into 9 areas. Area 0 is the central area corresponding to the grasp and the position of the actuators. The rest of the drawing is divided equally into 3 areas on each side of area 0 . Areas numbered 4 refer to an end-point out of the long handle. It is worth noting that the short handle length corresponds to area 0 and both areas denoted 1 . The direction and the number of the area pointed by the participant is recorded.

\section{B. Stimuli}

First, the actuators of both handles were tuned to provide equal intensity.

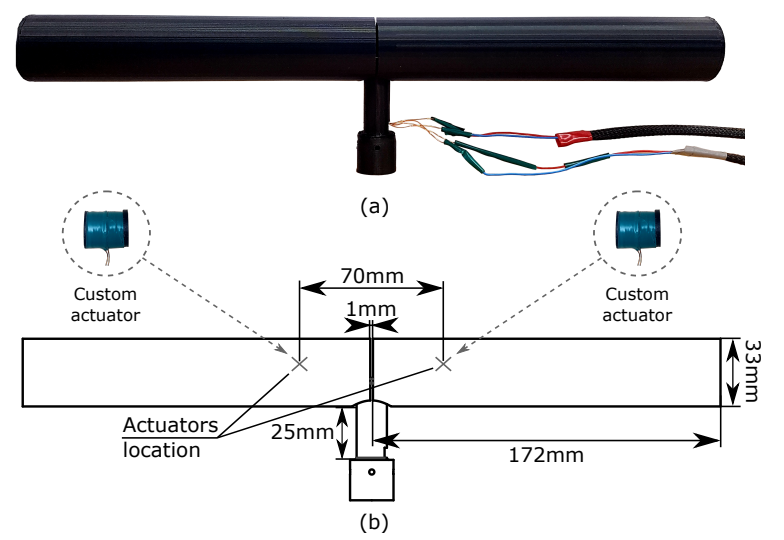

Fig. 2. (a) A picture of the long handle. (b) The long handle sagittal section.

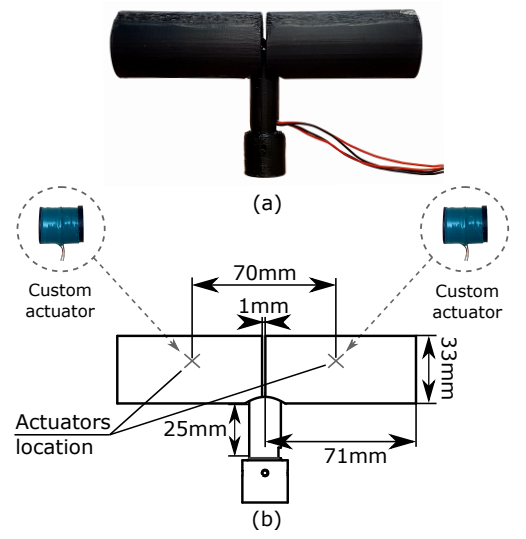

Fig. 3. (a) A picture of the short handle. (b) The short handle sagittal section.

Tactile stimuli corresponding to the PS as described in [5] were synthesized. Seven PS durations were chosen: $\{0.25$, $0.5,0.75,1,1.5,2$ and $2.5 \mathrm{~s}\}$. In total, 14 PS were synthesized, with seven different durations for each direction (forward or backward).

As explained in [5], the envelope of the two signals of a PS must not be linear as it would be detrimental to the illusion as the total perceived amplitude of the two signals would not be constant.

Here, the chosen envelope for all the signals, is an arc-tangent function. This function is like the logarithmic function and gives a reliable illusion. All envelopes were normalized in order to have the same maximal amplitude across all durations. The carrier signal is a sawtooth signal of $80 \mathrm{~Hz}$ filtered using a 1st order Butterworth with a cutting frequency of $40 \mathrm{~Hz}$. It was chosen to provide a well perceived illusion.

An example of a stimulus showing the two signals used to drive the two actuators for the $0.25 \mathrm{~s}$ duration is shown in Figure 5. One should notice that the envelope of the shown signal, chosen to clearly expose the carrier signal, has also an arc-tangent envelope. Its shape looks linear due to its short duration. 


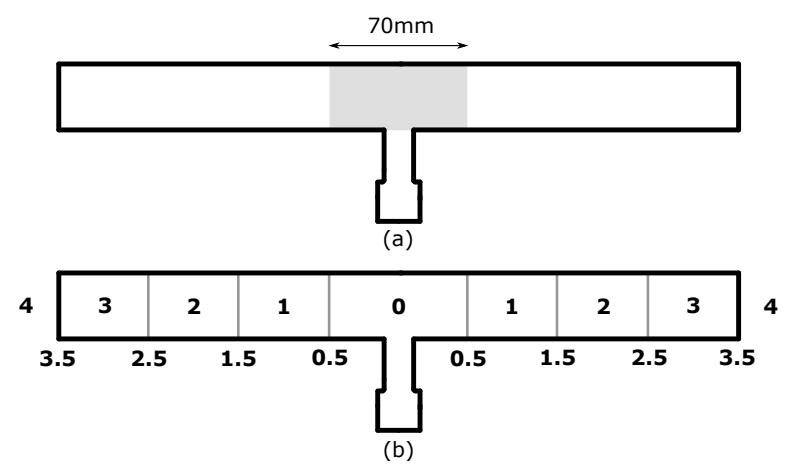

Fig. 4. (a) Drawing showed to the participants. The gray area represents the hand palm contact. (b) Drawing used by the experimenter.

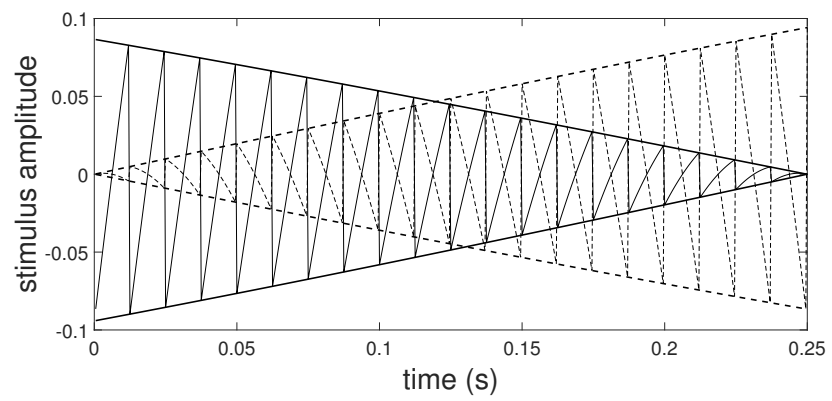

Fig. 5. Example of a signal generated. This signal was used for the phantom sensation of a duration of $0.25 \mathrm{~s}$. The full lines represent the signal generated and sent to the actuator 1 (envelope and carrier signal) and the dashed lines represent the signal generated and sent to the actuator 2 (envelope and carrier signal). The envelope is an arc-tangent function.

\section{Participants}

a) Experiments 1 and 2: 12 subjects (ten male, two female, with ages ranging from 23 to 28 years old) participated voluntarily in experiments 1 and 2.

b) Experiment 3: Five subjects (three male, two female, with ages ranging from 24 to 29 years old) participated voluntarily in experiment 3 .

None of the participants who took part in the experiment reported any motor, sensory or neurological disorders. They were unaware of the aim of the study, and had given their informed written consent.

\section{Experiments and testing conditions}

Subjects sat comfortably and held the handle horizontally by the middle with their right hand. The handle was maintained along their sagittal plane.

Subjects wore headphones with pink noise and closed their eyes during the experiment to avoid any inter-modality bias. The lights in the experimental room were dimmed. They received no feedback during the experiments. At the end of the experiments, an informal discussion took place with each participant to record their general impressions. These impressions concerned the clarity of the information, the perceived speed of the motion and whether they felt a relationship between the duration of the illusion and the distance travelled or any comment they thought relevant. Each experiment lasted approximately 20 minutes.
1) Experiment 1 - Discrimination of the direction: It was conducted in order to check if the duration influences the direction detection. In this experiment, the long handle has been used. In order to avoid fatigue, only three durations have been tested: the shortest $(0.25 \mathrm{~s})$, the longest $(2.5 \mathrm{~s})$ and one in-between (1 s).

A two-alternative forced choice protocol was used to validate the discrimination of the direction of the PS. For each duration, pairs of forward/backward, backward/forward, forward/forward, and backward/backward have been prepared. This makes a total of 4 pairs for each duration, hence 12 pairs for all the tested durations. Each pair was repeated 5 times in a randomized order leading to a total of 60 pairs of signals per participant. For each pair of signals presented, the subject was asked to report which signal was going forward. The answer was recorded.

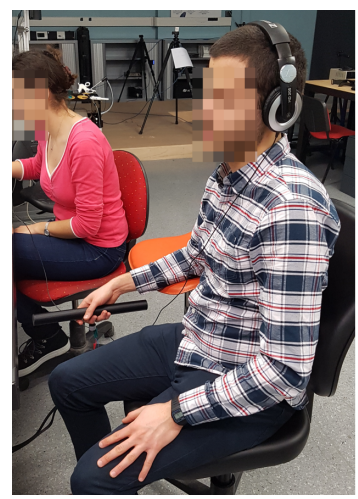

(a)

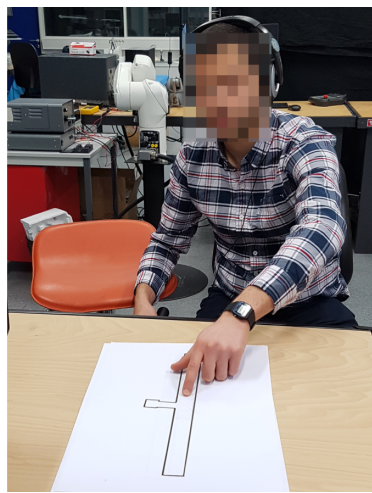

(b)
Fig. 6. Participant performing the (a) Experiment 1 (b) Experiment 2 or 3.

2) Experiment 2 - Out of hand detection: In this experiment, the long handle was used. Participants saw the handle. The drawing placed in front of them is shown in Fig. 4(a). As explained in section II-A, the drawing is a $1: 1$ representation of the long handle. It was explained to the participants that the gray area represented their grasp on the handle.

Participants were presented stimuli and informed of their direction and were asked for each to locate the end of the movement on the sheet of paper in front of them. After each stimulus, the participant answer was recorded by the experimenter using the areas presented in II-A and shown in Fig. 4(b). The direction of motion has also been recorded.

Each of the 14 stimuli described in II-B was presented five times in a random order leading to 70 randomized trials separated by rest periods.

3) Experiment 3 - Out of object detection: In this experiment, the short handle was used although the drawing used in experiment 2 was presented to the participants. Participants did not see the handle. This experiment was conducted to test whether the end-point could be out of the handle. The difference between the drawing and the object was chosen in order to avoid biasing participants into choosing an end-point in the handle. 
Otherwise, the experimental procedure was the same as in Experiment 2.

\section{DATA RECORDING, ANALYSIS AND RESULTS}

The data collected during our experiments did not have a normal distribution. For this reason, medians and interquartile ranges were used to represent them. Non-parametric tests were also used for the statistical analyses.

1) Experiment 1 - Discrimination of the direction: Fig. 7 shows a box plot of the direction detection probability. 1 and 0 represent, respectively, the probability of detection of the direction $100 \%$ and $0 \%$ of the time. In this plot, all data from all participants are pooled together. A Friedmann test showed that there is no significant difference between the three durations $(p>0.05)$.

The duration may not affect the probability of direction detection of a PS.

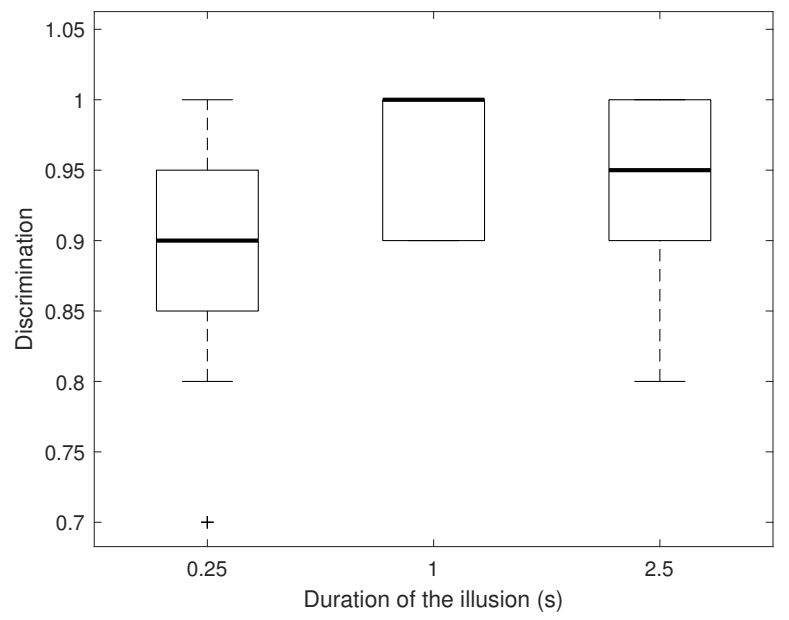

Fig. 7. Experiment 1 - Box plot of the probability of discrimination of the direction depending on the duration of the illusion. The diagram shows the median observation. The lower and upper quartiles are also represented. Data falling outside the interquartile range are plotted as outliers of the data.

2) Experiment 2 - Out of hand detection: As explained in II-D, after the pointing of the participant on the sheet depicted in Fig. 4(a), the experimenter records the given answer based on the drawing presented in Fig. 4(b), as well as the direction of the motion.

Area boundaries $(0.5,1.5,2.5$ and 3.5) have been used to facilitate the data collection. To simplify the analysis, these boundaries have been merged with the adjacent area. The forward and backward directions have been also pooled together.

Data have been separated into three groups:

- Participants who perceived the end-point of the stimulation both in and out of their hand. This group comprises seven out of twelve participants.

- Participants who perceived the end-point of the stimulation mostly or only in their hand (only the areas number

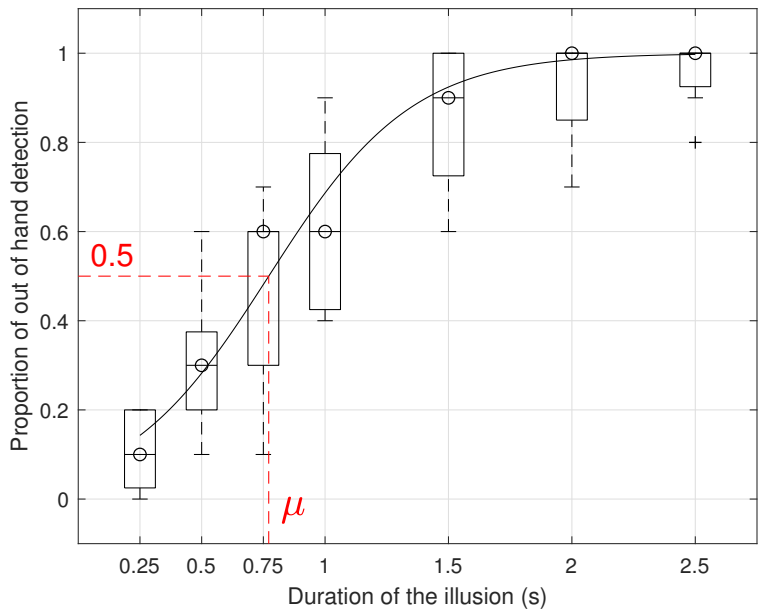

Fig. 8. Experiment 2 - Psychometric curve of the detection of the ending of the movement out of the hand as a function of the duration of the illusion. The median data for all participants who perceived in and out of hand movement, represented with boxplots, were used to compute this psychometric curve. The point of out hand of hand perception $\mu=0.77 \mathrm{~s}$ is plotted.

0 and 0.5 are reported). This group comprises three out of twelve participants.

- Participants who perceived the end-point of the stimulation mostly or only out of their hand (only areas 1 or bigger are reported). This group comprises two out of twelve participants.

For each group, a psychometric curve was fitted to the data. For the first group, the psychometric curve is drawn in Fig. 8. For the two other groups the curves are drawn in Fig. 9. The point of out of hand perception, $\mu$, is the duration at which the psychometric curve crosses a probability of 0.5 . For the first group, reporting in and out of hand perception, $\mu=0.77 s$.

Fig. 10 represents a heat map of the percentage of detection in an area depending on the duration. As explained, the hand corresponds to the area 0 of the drawing hence a detection in the area 1 or above is not in the hand. It was computed for all participants of the three groups pooled together. The heat map seems to suggest that there is a relationship between the duration and the perceived area containing the end-point of the movement.

The end-point of the movement was perceived $67 \%$ of the time out of the hand. The end-point of the movement was perceived $48 \%$ of the time in the areas marked 1 . These areas are close to the hand but out of the grasp.

3) Experiment 3 - Out of object detection: One subject out of five was discarded because the participant explained after the experiment that his/her answers were not based on the perceived area but rather on a mental correspondence between the size of the drawing and the used handle.

Figure 11 shows the psychometric curve for the answers pooled across the four retained participants. The point of out of object perception, $\mu$, is the duration at which the psychometric curve crosses a probability of $0.5, \mu=1.5 \mathrm{~s}$ 


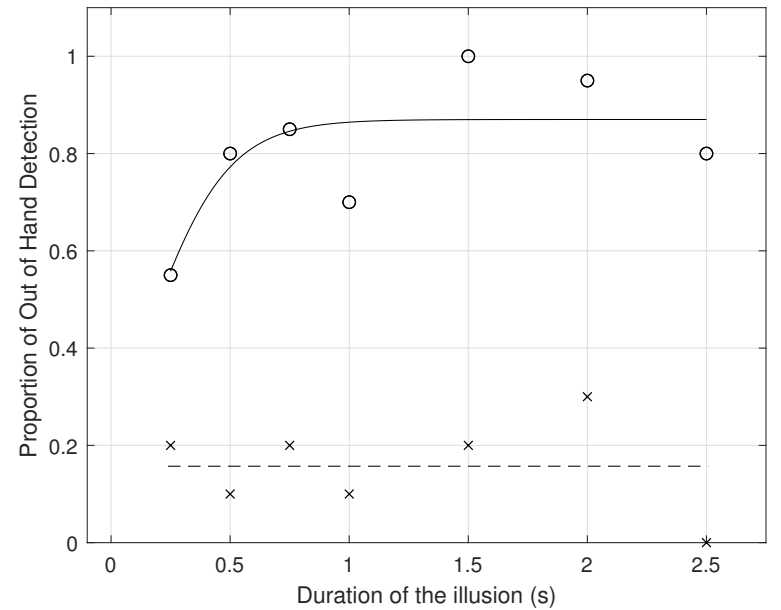

Fig. 9. Experiment 2 - Psychometric curve of the detection of the endpoint of the movement out of the hand as a function of the duration of the illusion for all participants who perceived mostly in or mostly out of hand movement. Areas $0.5,1.5,2.5$ and 3.5 were grouped with areas $0,1,2$ and 3 respectively. The two directions were treated together. The full line and 'o' correspond to out of hand participants. The dashed line and ' $x$ ' correspond to in hand participants.

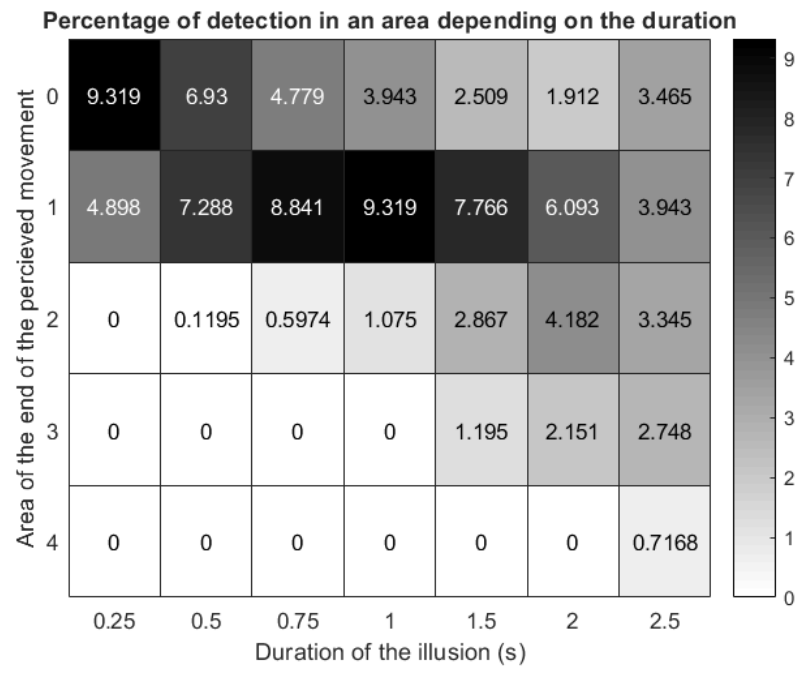

Fig. 10. Experiment 2 - heat map of the percentage of detection in an area depending on the duration for all participants. Areas 0.5, 1.5, 2.5 and 3.5 were grouped with Areas $0,1,2$ and 3 respectively. The two directions were treated together.

for out of object perception.

Fig. 12 represents the heat map of the percentage of detection in an area depending on the duration. It was computed for the retained participants. As explained, the handle size corresponds to the areas 0 , and both 1 . Hence a detection in the area 2 or above is considered out of the handle.

Participants' answers have been processed like in Experiment 2.

The heat map seems to suggest that there is a relationship between the duration and the perceived area containing the end-point of the movement.

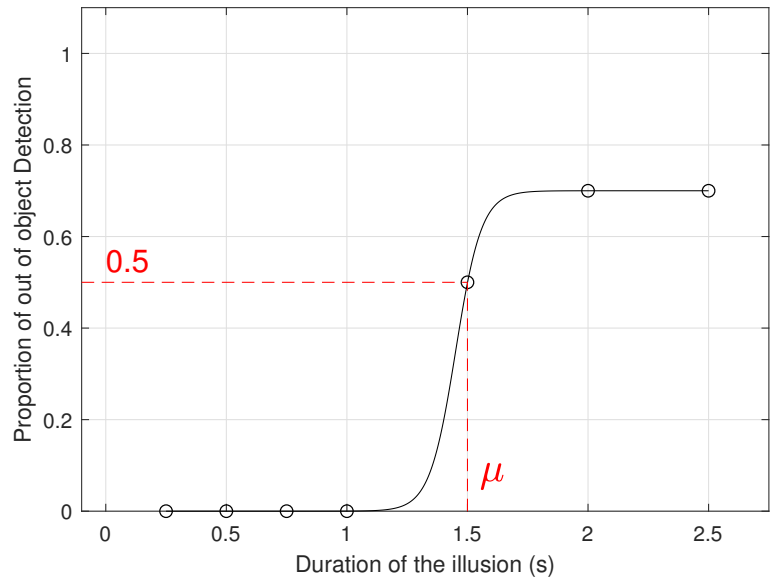

Fig. 11. Experiment 3 - Psychometric curve of the detection of the endpoint of the movement out of the object as a function of the duration of the illusion. The median of the data of the four retained participants were used to compute this curve. The two directions were treated together. The 'o' corresponds to the median values. The point of out of object perception $\mu=1.5 \mathrm{~s}$ is plotted.

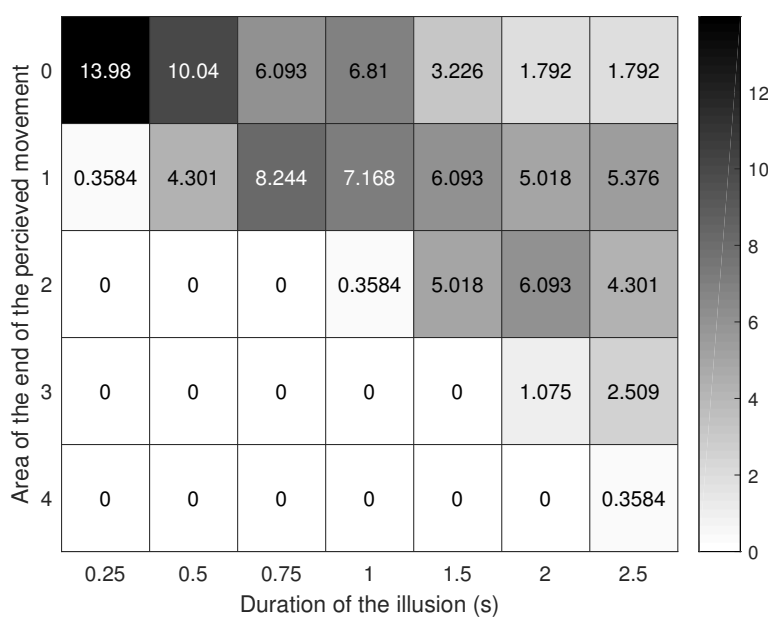

Fig. 12. Experiment 3 - Percentage of detection in an area depending on the duration for the four retained participants. Areas 0.5, 1.5,2.5 and 3.5 were grouped with Areas 0,1,2 and 3 respectively. The two directions were treated together.

The end-point of the movement was perceived almost $20 \%$ of the time out of the hand. It was perceived $16 \%$ of the time in the areas 2 . These areas are close to the handle but out of it.

\section{DISCUSSION}

Experiment 1 shows that the duration has no effect on direction discrimination for durations between 0.25 and $2.5 s$.

Experiment 2 shows that if the duration of the PS is greater than $\mu=0.77 s$, then the end-point of the motion is perceived out of the hand, in the handle.

Experiment 3 shows that if the duration of the PS is greater than $\mu=1.5 s$, then the end-point of the motion is perceived out of the handle, in the air.

Experiment 2 shows that for some participants, the end of the motion is either always in the hand or always out of the 
hand.

A. Mechanisms likely responsible for the out of hand and out of object perception

Participants perceived the end-point of the PS out of their hand or out of the object depending on the duration. A likely explanation of this perception relies on the process of the evaluation of distance and motion. This process is a combination of different factors. First, personal experiences are accumulated through all our contact with moving objects [13]. Secondly, our perception may be described as a weighted average of spatial and temporal judgments. This accumulation forms what is called a "natural context" of perception of distance and motion [14]. PS is a case of spatiotemporal interaction that seems to fit in the model described by Nguyen [13] and Jones [14]. An increased duration in a constant-velocity movement will be perceived as an increased distance travelled in this "natural context". The heat maps presented show a likely relation between the duration and the end-point position. The duration $\mu$ from which the end-point is out of the hand is shorter than the duration $\mu$ from which the end-point is out of the handle. These results support the idea that a bigger duration is perceived as a bigger travelled distance in a constant velocity context. Perception of distance is also not only based on the sensory information but on the mental scheme of one's body in the world [15]. The handle is probably added to the body mental scheme [10] [16], resulting in a possible perception out of the hand/handle. Participants also felt out of handle end-points. Out of the handle end-points may have been in the continuity of the movement and not rejected because the handle was hidden making the felt position more likely.

\section{B. Mechanisms likely responsible for the only in hand per- ception}

Some participants (three out of the 12) of the experiment 2 felt only in hand end-points. They all explained in the postexperiment discussion that they felt different speeds of motion. They explained that the longer the durations, the slower they were perceived. In the same way, shorter durations were perceived as faster. It is thus likely that a long duration was not perceived as a long distance since they perceived a change of speed of motion. Nguyen et al. in [13] explain that localisation depends on velocity perception. They also explain that a "compressive mislocalisation" is possible and depends on the velocity. This "compressive mislocalisation" would appear here as only in hand perception.

\section{Mechanisms likely responsible for the out of hand per- ception}

Two participants out of the 12 of the experiment 2 felt only out of hand perception and rarely felt the end-point in their hand. The psychometric curve of out of hand detection as a function of duration is not constant. It is possible that those participants overestimated the traveled distance with out of hand end-points as a result.

\section{Some of the study limits}

A tactile interface to record the exact position of the end-point of the movement could have been used. Besides, increasing the number of participants should make our observations more reliable. In this study we did not take into account the direction of motion. In [13], it is reported that there is a difference of distance perception depending on the direction. Therefore, we should reprocess our data as a function of the direction of the PS.

\section{CONCLUSION}

This study shows that an increase in duration of a Phantom Sensation results in a perceived end-point of the motion out of the hand or out of the object. Furthermore, the results suggest that a Phantom Sensation depending on its duration is not bound by the position of the actuators. In the near future, a precise measurement device will be added to our experimental setup to let us further investigate the effect of speed and direction, in order to provide reliable results.

\section{REFERENCES}

[1] H. Burtt, "Tactual illusions of movement," Journal of experimental Psychology, vol. 2, no. 5, pp. 371-385, 1917.

[2] V. Hayward, "Tactile illusions," Scholarpedia, vol. 10, no. 3, p. 8245, 2015, revision \#151585.

[3] C. Sherrick and R. Rogers, "Apparent haptic motion," Perception \& Psychophysics, vol. 1, pp. 175-180, 1966.

[4] F. Geldard and C. Sherrick, "The cutaneous rabbit: A perceptual illusion," Science, vol. 178, 1972.

[5] D. Alles, "Information transmission by phantom sensations," IEEE Transactions on Man-Machine Systems, vol. 11, no. 1, pp. 85-91, 1970.

[6] J. V. S. Luces, K. Okabe, Y. Murao, and Y. Hirata, "A phantomsensation based paradigm for continuous vibrotactile wrist guidance in two-dimensional space," IEEE Robotics and Automation Letters, vol. 3, no. 1, pp. 163-170, Jan 2018.

[7] E. Gardner and B. Sklar, "Discrimination of the direction of motion on the human hand: a psychophysical study of stimulation parameters," Journal of Neurophysiology, vol. 71, no. 6, pp. 2414-2429, 1994.

[8] R. Cholewiak, "The perception of tactile distance: Influences of body site, space, and time," Perception, vol. 28, pp. 851-875, 1990.

[9] C. Berger and M. Gonzalez-Franco, "Expanding the sense of touh outside the body," in ACM Symposium on Applied Perception, 2018.

[10] M. Miyazaki, M. Hirashima, and D. Nozaki, "The cutaneous rabbit hopping out of the body," Journal of Neuroscience, vol. 30, no. 5, pp. 1856-1860, February 2010.

[11] M. Eimer, B. Forster, and J. Vibell, "Cutaneous saltation within and across arms: A new measure of the saltation illusion in somatosensation," Perception \& Psychophysics, vol. 67, no. 3, pp. 458-468, 2005

[12] H.-Y. Yao and V. Hayward, "Design and analysis of a recoil-type vibrotactile transducer," The Journal of the Acoustical Society of America, vol. 128, no. 2, pp. 619-627, 2010. [Online]. Available: https://doi.org/10.1121/1.3458852

[13] E. Nguyen, J. Taylor, J. Brooks, and T. Seizova-Cajic, "Velocity of motion across the skin influences perception of tactile location," Journal of Neurophysiology, vol. 115, pp. 674-684, 2016.

[14] B. Jones and Y. Huang, "Space-time dependencies in psychophysical judgment of extent and duration: Algebraic models of the tau and kappa effects." Psychological Bulletin, vol. 91, no. 1, pp. 128-142, 1982.

[15] S. Badde and T. Heed, "Towards explaining the spatial touch perception: Weighted integration of multiple location codes," Cognitive Neuropsychology, vol. 33, no. 1-2, pp. 26-47, 2016.

[16] L. Miller, L. Montroni, E. Koun, R. Salemme, V. Hayward, and A. Farné, "Sensing with tools extends somatosensory processing beyond the body." Nature, vol. 561, no. 7722, pp. 239-242, 2018. 